\title{
Astaxanthin profiles and corresponding colour properties in Australian farmed black tiger prawn (Penaeus monodon) during frozen storage
}

\author{
Kent J Fanning, Carl Paulo, Sharon Pun, Caterina Torrisi, Kerrie Abberton, Paul Exley \& \\ Sue Poole \\ Department of Agriculture, Fisheries and Forestry, Crop and Food Science, Agri-Science Queensland, 39 Kessels Rd, \\ Coopers Plains, Queensland 4108, Australia
}

Correspondence: K Fanning, Department of Agriculture, Fisheries and Forestry, P.O. Box 156, Archerfield BC, Queensland 4108, Australia. E-mail: kent.fanning@daff.qld.gov.au

\begin{abstract}
The colour of commercial cooked black tiger prawns (Penaeus monodon) is a key quality requirement to ensure product is not rejected in wholesale markets. The colour, due to the carotenoid astaxanthin, can be impacted by frozen storage, but changes in colour or astaxanthin profile, during frozen storage, have not been studied in detail. Subsequently in this study, the aims were to define the astaxanthin (as cis, trans, mono-ester and di-ester forms) content, together with the colour properties, in both pleopods (legs) and abdominal segments. Changes in astaxanthin content and colour properties were further determined during frozen storage $\left(-20^{\circ} \mathrm{C}\right)$. Total astaxanthin content was seen to decrease in all samples over time, with the rate of degradation being significantly greater $(P<0.05)$ in pleopods than abdomen. In both pleopods and abdomen, rate of degradation of esterified forms was significantly greater $(P<0.05)$ than non-esterified forms. Hue angle (increase), $a^{*}$ value (decrease) and $L$ value (increase) were all seen to significantly change $(P<0.05)$ during storage, with changes being more prevalent in the pleopods. The pleopods are the key indicator of astaxanthin and colour loss in cooked black tiger prawns and preservation strategies are required to preserve astaxanthin and colour during frozen storage.
\end{abstract}

Keywords: prawns, colour, astaxanthin, frozen storage

\section{Introduction}

Prawn farming has grown to be the fourth largest aquaculture sector in Australia, by quantity and value, behind salmonids, tuna and oysters (ABARE 2010). Total production volume of farmed prawns is currently 5381 tonnes yielding a revenue return to the industry of A $\$ 77.5$ million, with Penaeus monodon (black tiger prawn) being the most cultivated species (ABARE 2010). Australian prawn aquaculture ventures are focused in Queensland, a tropical region, with open pond grow-out systems. Production is typically presented as cooked product, both chilled and frozen, into the domestic market.

Cooked black tiger prawns are characteristically red-orange in colour and the depth of colour is considered a primary quality attribute driving consumer acceptance and price attained (Fujii, Nakashima, Hashidzume, Uchiyama, Mishiro \& Kadota 2010; Parisenti, Beirão, Tramonte, Ourique, da Silveira Brito \& Moreira 2011b). Where prawns do not meet the consumer colour expectation for this species, substantial revenue loss occurs from consequent rejection at retail markets (Aqua Marine Marketing, Kippa-Ring, Queensland, 2010 and Australian Prawn Farmers Association, 2011, personal communication). The red-orange pigmentation in black tiger prawns is associated with the carotenoid astaxanthin (3,3'-dihydroxy- $\beta$, $\beta$-carotene- $4,4^{\prime}$-dione), present in the exoskeleton and epidermal layers of the prawn (Menasveta, Worawattanamateekul, Latscha \& Clark 1993; 
Tume, Sikes, Tabrett \& Smith 2009). Astaxanthin is accumulated through ingestion and dietary sources which include both environmental algal carotenoids present in the pondwater and the applied prawn feed (Chien \& Jeng 1992; Boonyaratpalin, Thongrod, Supamattaya, Britton \& Schlipalius 2001).

The effects of prawn maturity and feeding regimes on astaxanthin content and prawn colour have been previously studied in farmed prawns, including Penaeus monodon (Menasveta et al. 1993; Okada, Nureborhan \& Yamaguchi 1994; Boonyaratpalin et al. 2001) and Litopenaeus vannamei (Ju, Deng, Dominy \& Forster 2011; Parisenti, Beirao, Maraschin, Mourino, Do Nascimento Vieira, Bedin \& Rodrigues 2011a; Parisenti et al. 2011b). Recent studies have demonstrated the effect of environmental background colour on the mobilization of astaxanthin and transition between esterified and non-esterified forms of the carotenoid in live prawns (Tume et al. 2009; Wade, Anderson, Sellars, Tume, Preston \& Glencross 2012). However, there has been little published on colour and astaxanthin changes during post-harvest storage following cooking, with only three studies found on frozen Pandalus borealis (Bak, Andersen, Andersen \& Bertelsen 1999) and dried Penaeus indicus (Niamnuy, Devahastin, Soponronnarit \& Vijaya Raghavan 2008; Becerra, Flores, Valerio-Alfaro, Soto-Rodriguez, Rodriguez-Estrada \& Garcia 2014).

Within Australian prawn supply chains, cooked black tiger prawns are sometimes subject to long periods of frozen storage to ensure continuous availability of product. During such storage, it has been observed that the prawns lose the characteristic red-orange colouration and fade to a lighter, more yellow hue (Aqua Marine Marketing, Kippa-Ring, Queensland 2010 and Australian Prawn Farmers Association, 2011, personal communication).

The present study determines colour and astaxanthin content during long-term frozen storage of black tiger prawns and highlights colour changes, along with astaxanthin degradation patterns. Furthermore, it is the first study to report astaxanthin content and colour of the pleopods (legs) specifically.

\section{Materials and methods}

\section{Chemicals}

Hexane (Ajax, Taren Point, New South Wales, Australia) and ethanol (Ajax) were analytical grade, and methyl tert-butyl ether (Burdick \& Jackson, Muskegon, MI, USA), methanol (Mallinckrodt, Phillipsburg, NJ, USA) and dichloromethane (Merck, Darmstadt, Germany) were HPLC grade. Trans-astaxanthin and astaxanthin dipalmitate were purchased from Carotenature (Lupsingen, Switzerland).

\section{Prawns for trials}

Black tiger prawns (Penaeus monodon) were sourced from five geographically separated commercial prawn farms in Queensland, Australia: two farms were located on the southern coast, two on the central coast and one on the northern coast of Queensland. Prawns were harvested, cooked and chilled under commercial operational practice on farm and were then delivered on ice to the laboratory facility by refrigerated transport at 1-2 days post harvest. On arrival, prawns were blast frozen to a core temperature of below $-20^{\circ} \mathrm{C}$. Prawns, $5 \mathrm{~kg}$ quantities, were stored in plastic-lined cardboard boxes at $-20 \pm 1^{\circ} \mathrm{C}$. All 0 day (baseline) sample analyses were assessed on chilled prawns shortly after arrival.

\section{Frozen storage trials}

Table 1 provides an overview of sampling and storage times within trials. In the preliminary trial, prawns were sourced from the northern farm in late June 2010. Six individual randomly selected prawns were analysed for astaxanthin content and colour properties at $0,12,14,22,28,56$ and 119 days storage. In subsequent trials, prawns were sourced from the five commercial farms during 2011.

Baseline data for colour properties and astaxanthin content was generated from day 0 prawn samples. On each sampling day, 20 randomly selected individual prawns were each measured for colour properties and then prawns were pooled to create a composite sample for astaxanthin content analysis. For the subsequent sampling of frozen stored prawns, 10 individual prawns were assessed for colour properties and then a pooled sample of the 10 prawns was composited for astaxanthin content at $7,28,56,124,187$ and 371 days storage.

\section{Sample preparation}

In the preliminary storage trial (119 days total storage), abdominal segments 2,4 and 6 were taken (full cross-section of intact shell and flesh 
Table 1 Summary of sampling and frozen storage times within trials

\begin{tabular}{lcc}
\hline & Preliminary trial $(\boldsymbol{n}=\mathbf{1})$ & Frozen storage trials $(\boldsymbol{n}=\mathbf{7})$ \\
\hline Region of prawn sampled & Abdomen - segments 2, 4, & Pleopods - segments $1-6$ \\
& & Abdomen - segments $2,3,4$ \\
Baseline samples (0 day) & $n=6$ individuals chromameter and astaxanthin analyses & $n=20$ individuals chromameter \\
& & $n=20$ composite astaxanthin \\
Samples during storage & $n=6$ individuals chromameter and astaxanthin analyses & $n=10$ individuals chromameter \\
& & $n=10$ composite astaxanthin \\
Storage time (days) & 1214222856119 & 72856124187371 \\
\hline
\end{tabular}

with pleopods removed) of six individual prawns at each time point and processed individually for analysis. For baseline samples and the seven additional storage trials (371 days total storage), abdominal segments 2, 3 and 4 (as a block, including full cross-section of intact shell and flesh, with pleopods removed) were taken. Pleopods associated with abdominal segments 1 through to 6 were removed and pooled as a separate assessment sample for comparison of astaxanthin content in these structures relative to the abdominal sections. This was undertaken as it was anticipated that the colour and astaxanthin profiles, and degradation rates of both colour and astaxanthin, would be different between the pleopods and abdomen. For the 0 day samples, abdominal segments of 20 prawns were pooled together and the pleopods were similarly pooled. For the $7,28,56,124,187$ and 371 days time points, of all seven storage trials, a reduced number of 10 prawns were used with abdominal segments and pleopods pooled separately as consistent results between composite samples of 20 and 10 randomly selected prawns were observed.

Pooled samples were diced, fed through a mincer and then cryogenically milled, to a fine powder, using a Retsch MM301 mill (Haan, Germany). The use of a composite sample for astaxanthin analysis was undertaken so as to allow direct correlation between colour measurement values attained and astaxanthin content for the same prawns. The coefficient of variation for the extraction and analysis method was determined as $<5 \%$ by undertaking six separate extractions on six aliquots of the same prawn pooled material, for both abdomen and pleopods.

\section{Extraction for astaxanthin analysis}

The extraction method was modified from a combination of previously described methods (Tume et al. 2009; Fanning, Martin, Wong, Keating, Pun \& O'Hare 2010). Ten millilitre acetone, containing $0.1 \%$ butylated hydroxyl-toluene (BHT), was added to $1 \mathrm{~g}$ (pleopod) or $5 \mathrm{~g}$ (abdominal segment) of the milled prawn sample and vortexed for 20 s. Samples were stored on ice throughout the extraction process. Five millilitre of $10 \% \mathrm{NaCl}$ (in water) and $10 \mathrm{~mL}$ of hexane were added followed by vortexing and centrifugation $\left(5000 \mathrm{~g}\right.$ for $3 \mathrm{~min}$, at $4^{\circ} \mathrm{C}$ ) to separate layers, with the top layer being transferred to another clean tube (hexane fraction). These steps were then repeated until the top layer was colourless. Combined hexane fractions were dried in a centrifugal evaporator at $25^{\circ} \mathrm{C}$ and then reconstituted in $2-10 \mathrm{~mL}$ of methanol/dichloromethane (50:50, v/v), containing 0.1\% BHT. Samples were filtered $(0.22 \mu \mathrm{m}$ syringe filter, Grace, Sydney, Australia) and placed into HPLC vials, before storing under nitrogen at $-80^{\circ} \mathrm{C}$ prior to HPLC analysis. Standard solutions were prepared similarly in methanol/dichloromethane (50:50, v/v), containing $0.1 \%$ BHT.

\section{HPLC analysis}

The HPLC system consisted of a SIL-10AD VP auto injector (Shimadzu, Kyoto, Japan), SCL-10A VP system controller (Shimadzu), LC-10AT VP liquid chromatograph (Shimadzu) and a SPDM10 A VP diode array detector (Shimadzu). Forty microlitre of each extract was injected onto a YMC C30 Carotenoid Column, $3 \mu \mathrm{m}$, $4.6 \times 250 \mathrm{~mm}$ (Waters, Milford, MA, USA), with a mobile phase consisting of $92 \%$ methanol: $8 \%$ $10 \mathrm{mM}$ ammonium acetate (phase A), and $100 \%$ methyl tert-butyl ether (phase B). The following $54 \mathrm{~min}$ gradient was used: $0 \mathrm{~min}$, $80 \%$ phase A; $48 \mathrm{~min}, 20 \%$ phase A; $49 \mathrm{~min}$, $80 \%$ phase A; $54 \mathrm{~min}, 80 \%$ phase A. 


\section{Identification and quantitation of astaxanthin forms}

Identification followed the LC-(APCI)MS method of Breithaupt (Breithaupt 2004), using the negative ion mode. The column, mobile phase and flow were as described above in HPLC Analysis.

Trans-astaxanthin was identified by comparison with the retention time and absorption spectra of the standard and mass spectra (negatively charged molecular ion $\mathrm{m} / \mathrm{z}=596.5$ (Breithaupt 2004)). Cis-astaxanthin peaks were identified by relative retention time, absorption spectra and mass spectra [negatively charged molecular ion $\mathrm{m} / \mathrm{z}=596.5$ (Breithaupt 2004)]. The two separate blocks of the various astaxanthin mono-esters and di-esters were identified by relative retention time (including comparison with astaxanthin dipalmitate standard), absorption spectra and mass spectra [mono-esters having backbone mass of $\mathrm{m} / \mathrm{z} 578.4$ (negatively charged molecular ion minus the fatty acid) (Breithaupt 2004)]

A trans-astaxanthin solution of approximately $2 \mu \mathrm{g} \mathrm{mL}^{-1}$ was made up in hexane and absorbance measured at $472 \mathrm{~nm}$. The concentration of solution was determined by the following formula, using $\mathrm{A}^{1 \%}$ of 1911 (Breithaupt 2004):

$$
\begin{aligned}
& \operatorname{Concentration}\left(\mu \mathrm{g} \mathrm{mL}^{-1}\right) \\
& =\text { Absorbance } \times 10000 / \mathrm{A}^{1 \%}
\end{aligned}
$$

The peak purity of each peak was determined. The actual concentrations of the standard solutions were then calculated by multiplying the concentration determined spectrophotometrically by the \% peak area of the standard peak as determined by HPLC. Standard curves were linear over the range 0.03 to $10 \mu \mathrm{g} \mathrm{mL}^{-1}$ with $r^{2}$ values of $<0.999$. The trans-astaxanthin standard curve was used to determine the concentration of the other astaxanthin forms. Total astaxanthin content was determined as the sum of cis-astaxanthin, trans-astaxanthin, mono-ester and di-ester content. Non-esterified astaxanthin was determined as the sum of cis-astaxanthin and trans-astaxanthin, whereas esterified astaxanthin was determined as the sum of mono-ester and di-ester content.

\section{Colour measurement}

Colour was measured using a Minolta Chroma meter CR-400 (Konica Minolta Sensing, Osaka,
Japan) fitted with a CR-A33b light projection tube. The instrument was calibrated using a CR-A43 white calibration plate (illuminant $\mathrm{C}: Y=88.5$, $x=0.3157, y=0.3224$ ) at the beginning of each sampling time point. For this study, the CIELAB colour space $\left(\mathrm{L}^{*} \mathrm{a} \mathrm{b}^{*}\right)$ was used. Each prawn had a total of six colour measurements taken of the abdomen and an additional six of the pleopods. Abdominal segments 2, 4 and 6 or segments 2, 3 and 4 were measured for colour as described above in sample preparation. A single averaged measurement from the three segments of both left and right sides was recorded. To measure colour of pleopods, prawn was curled to gather pleopods to a centralized point. Triplicate measurements were averaged from both left and right pleopods and recorded. Results reported were averaged from all prawns during each sampling time. Values for chroma and hue angle were calculated by the following formula:

$$
\begin{gathered}
\text { Chroma }=\left(\mathrm{a}^{* 2}+\mathrm{b}^{* 2}\right)^{1 / 2} \\
\text { Hue angle }=\tan ^{-1}\left(\mathrm{~b}^{*} / \mathrm{a}^{*}\right)
\end{gathered}
$$

\section{Data and statistical analysis}

Results are presented as mean \pm standard deviation (SD) unless otherwise specified, except for Fig. 1b where mean \pm standard error of the mean (SEM) was used for clarity of the figure. Differences in astaxanthin concentration and colour properties between storage times were evaluated using one-way analysis of variance (ANOvA) and the Tukey HSD procedure using JMP software (Version 7). Both linear and non-linear regression analysis was undertaken using SIGMAPLOT (Version 10).

Astaxanthin loss during storage has been shown to follow first-order reaction kinetics, in dried prawn, as defined by $\ln (C / C o)=-k t$; where $C$ is astaxanthin content at time point of interest, $\mathrm{Co}$ is initial astaxanthin content, $k$ is the rate constant and $t$ is time (Niamnuy et al. 2008). In (C/Co) was plotted against time to calculate $k$, using SIGMAPLOT.

\section{Results}

In the preliminary trial (Table 1), the total astaxanthin content of abdominal segments decreased over time during the 119 days of frozen storage (Fig. 1a). Changes in total astaxanthin content 

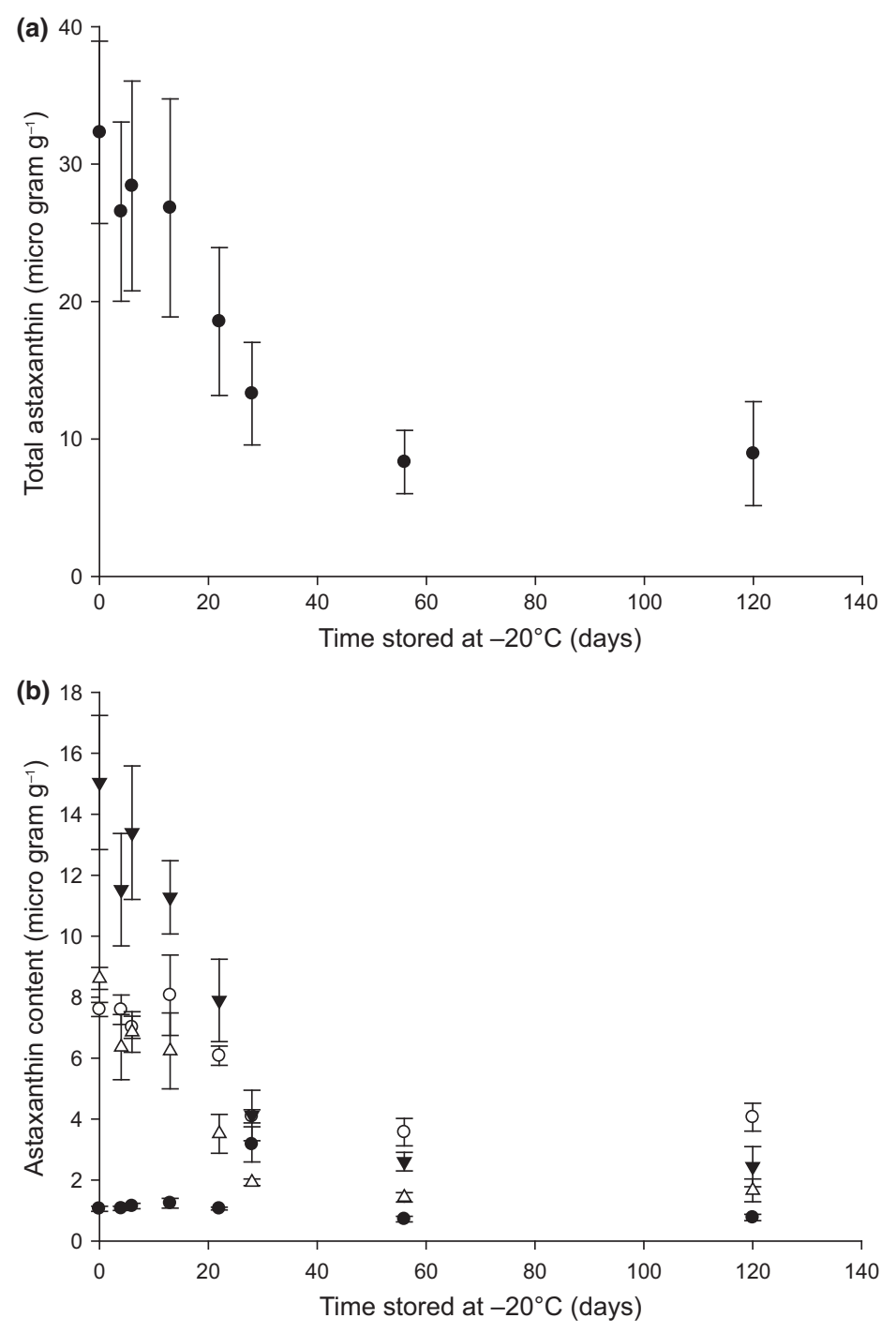

Figure 1 Change in total astaxanthin content (a, mean $\pm \mathrm{SD}$, $n=6$; asterisk indicates value is significantly different $[P<0.05]$ to 0 day) and content of astaxanthin forms (b, • - cis-astaxanthin, o trans-astaxanthin, $\boldsymbol{\nabla}-$ monoester, $\Delta$ - di-ester; mean \pm SEM [SEM used rather than SD for clarity], $n=6$ ) in abdominal segments of prawns during storage at $-20^{\circ} \mathrm{C}$ for up to 119 days.

were significant $(P<0.007)$ from 22 days onwards when compared to initial levels (0 day), with mean losses of total astaxanthin of $60 \%$ after 28 days, $87 \%$ after 56 days and $86 \%$ after 119 days. All four forms of astaxanthin decreased with time (Fig. 1b) with a significantly greater $(P<0.006)$ degradation rate for mono-ester astaxanthin $(k=3.61 \pm$ $1.30 \mathrm{~s}^{-1}, \times 10^{2}$, over 56 days) and di-ester astaxanthin $\quad\left(k=3.27 \pm 1.36 \mathrm{~s}^{-1}, \quad \times 10^{2}, \quad\right.$ over 56 days) than trans-astaxanthin $(k=1.19 \pm 0.58$ $\mathrm{s}^{-1}, \times 10^{2}$, over 56 days) and cis-astaxanthin $\left(k=0.66 \pm 0.67 \mathrm{~s}^{-1}, \times 10^{2}\right.$, over 56 days). This resulted in a shift from a predominance of esterified forms $(74 \%$ of total astaxanthin at 0 day) to nonesterified forms $(54 \%$ of total astaxanthin at 119 days) with time. Both the $\mathrm{L}^{*}$ value and hue angle were significantly higher at 119 days when compared with initial values $(P<0.05$, data not shown).

The total astaxanthin content present in the prawns from the various locations (Table 1) ranged from 5.5 to $11.5 \mu \mathrm{g} \mathrm{g}^{-1}$, in the abdomen, and from 47.0 to $159.7 \mu \mathrm{g} \mathrm{g}^{-1}$, in the pleopods (Table 2). In the abdominal segments, the mean content of trans-astaxanthin and mono-ester astaxanthin were similar, but significantly higher than di-ester astaxanthin $(P<0.03)$ and cis-astaxanthin $(P<0.0001)$ (Table 3). In the pleopods, mean mono-ester astaxanthin, di-ester astaxanthin and trans-astaxanthin content were not significantly different from each other, but were all significantly greater than cis-astaxanthin content $(P<0.007)$.

For the 371 days frozen storage trials (Table 1), there were significant changes $(P<0.05)$ in some 
of the colour properties of the abdominal segments and pleopods during storage with changes generally being greater in the pleopods (Fig. 2). In the abdominal segments, significant increases in $\mathrm{L}^{*}$ value (becoming lighter) were seen following 371 days in four of seven trials, significant decreases $(P<0.05)$ in $a^{*}$ value were evident following 371 days in two of seven trials, and significant increases $(P<0.05)$ in hue angle (shift from red/orange to orange/yellow) were evident following 371 days in two of seven trials. Chroma and $b^{*}$ value were generally constant.

In the pleopods, significant increases $(P<0.05)$ in $L^{*}$ value were evident following 371 days in two of seven trials. However, in all seven trials, significant decreases $(P<0.05)$ in $\mathrm{a}^{*}$ value, significant decreases in chroma, and significant increases in hue angle, were observed following 187 and 371 days. The $b^{*}$ value was generally constant only decreasing significantly $(P<0.05)$ in 1 trial after 371 days.

Total astaxanthin content decreased with time, in both abdominal segments and pleopods during $-20^{\circ} \mathrm{C}$ storage in the 371 days trials (Fig. 3). The rate of astaxanthin degradation was significantly greater $(P<0.05)$ in the pleopods than the abdominal segments (Table 4). The astaxanthin degradation followed first-order kinetics up to 187 days but for 371 days departed from firstorder relationship. There was a directly proportional relationship between the rate of astaxanthin loss, over 187 days, and initial astaxanthin content in the abdomen $\left(r^{2}=0.86\right)$ and pleopods $\left(r^{2}=0.97\right)$.

Total astaxanthin versus $L^{*}$ value, $a^{*}$ value and hue angle (for the combined data from all 7 of the 371 days trials, when fitted using an exponential decay function) showed poor to moderate correlations for the pleopods $\left(r^{2}=0.34\right.$ for $\mathrm{L}^{*}, r^{2}=0.37$ for $\mathrm{a}^{*}, r^{2}=0.46$ for hue) and abdominal segments $\left(r^{2}=0.35\right.$ for $\mathrm{L}^{*}, r^{2}=0.18$ for $\mathrm{a}^{*}, r^{2}=0.41$ for hue). For the pleopods, correlations from the individual trials were better than the combined data. For example, for total astaxanthin versus hue angle the mean $r^{2}$ for the seven trials was $0.67 \pm 0.15$, with five of the trials having $r^{2}>0.71$, and, for total astaxanthin versus chroma the mean $r^{2}$ for the 7 trials was $0.60 \pm 0.20$. Analysis of baseline samples (0 day) of the storage trials yielded good correlations between total astaxanthin and hue angle $\left(r^{2}=0.69\right.$, fitted using exponential function) and 
total astaxanthin and $\mathrm{L}^{*}$ value $\left(r^{2}=0.70\right.$, fitted using exponential function), for the abdominal segments. However for the pleopods, there was no correlation between the initial astaxanthin content

Table 3 Mean astaxanthin content $\left(\mu \mathrm{g} \mathrm{g}^{-1}\right)$ and astaxanthin form as a percentage mean of total astaxanthin (in parentheses), present in black tiger prawns sourced from prawn farms in the major production areas of Queensland (mean \pm SD, $n=10$ )

\begin{tabular}{|c|c|c|}
\hline $\begin{array}{l}\text { Astaxanthin } \\
\text { form }\end{array}$ & $\begin{array}{l}\text { Abdominal } \\
\text { segments } \\
\mu \mathrm{g} \mathrm{g}^{-1}(\%)\end{array}$ & Pleopods $\mu \mathbf{g ~ g}^{-1}(\%)$ \\
\hline Cis-astaxanthin & $0.6 \pm 0.2^{c}(7 \pm 0.8)$ & $4.6 \pm 1.8^{\mathrm{B}}(5 \pm 1)$ \\
\hline Trans-astaxanthin & $3.4 \pm 1.0^{\mathrm{a}}(39 \pm 8)$ & $17.9 \pm 4.3^{A}(22 \pm 6)$ \\
\hline Mono-esters & $3.2 \pm 1.2^{\mathrm{a}}(35 \pm 7)$ & $47.5 \pm 26.3^{A}(46 \pm 7)$ \\
\hline Di-esters & $1.7 \pm 0.6^{b}(19 \pm 4)$ & $25.7 \pm 12.3^{A}(26 \pm 5)$ \\
\hline
\end{tabular}

Values in the same column that are followed by different letters are significantly different $(P<0.05)$. and hue angle or a* value but there was between initial astaxanthin content and $L^{*}$ value $\left(r^{2}=0.70\right.$, fitted using exponential function). No correlation was seen between total astaxanthin and chroma.

The content of each form of astaxanthin, in both abdominal segments and pleopods, decreased with storage at $-20^{\circ} \mathrm{C}$. There were significant differences $(P<0.05)$ between the degradation rate of each form of astaxanthin (Table 4). In both the abdominal segments and pleopods, degradation rate of mono-ester and di-ester forms were significantly greater $(P<0.05)$ than that of cis-astaxanthin and trans-astaxanthin. As with total astaxanthin, the degradation, of each form, followed first-order kinetics up to 187 days.

The differences in rate of degradation, of the various forms, resulted in a change in astaxanthin profile (as shown in Fig. 4), in both abdominal segments and pleopods. The predominance of
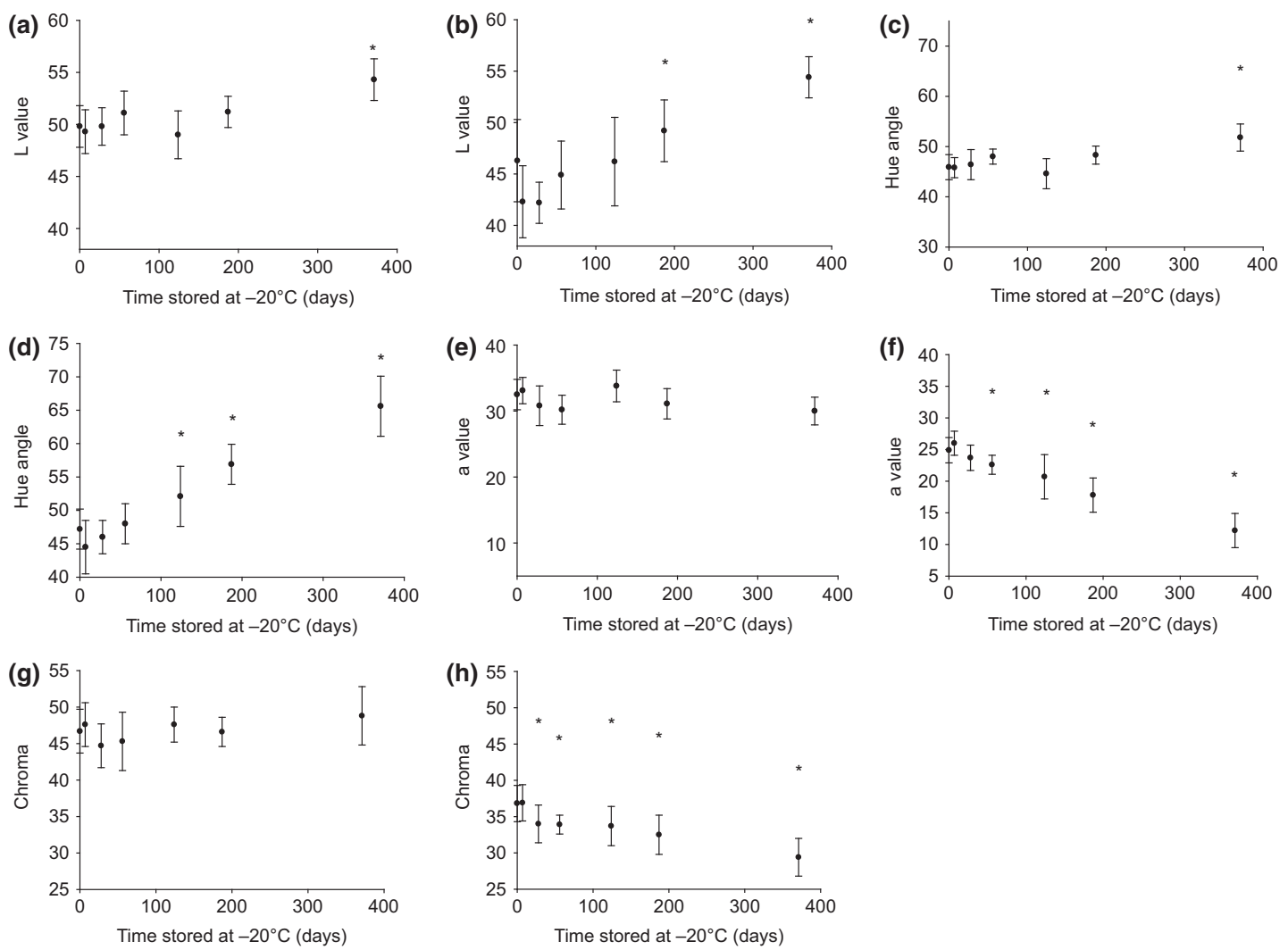

Figure 2 Change in $\mathrm{L}^{*}$ value (a - abdominal segment, $\mathrm{b}$ - pleopods), hue angle (c - abdominal segment, $\mathrm{d}$ - pleopods), $\mathrm{a}^{*}$ value (e - abdominal segment, $\mathrm{f}$ - pleopods) and chroma ( $\mathrm{g}$ - abdominal segment, $\mathrm{h}$ - pleopods) during $-20^{\circ} \mathrm{C}$ storage of prawns (mean $\pm \mathrm{SD}, n=20$ [0 day] or 10; data from single, representative 371 days trial shown; asterisk indicates value is significantly different $[P<0.05]$ to 0 day). 
esterified forms changed to a predominance of non-esterified forms. The $\%$ of cis-astaxanthin and trans-astaxanthin were significantly higher $(P<0.05)$ after storage for 371 days at $-20^{\circ} \mathrm{C}$, whereas the \% of mono-ester astaxanthin and di-ester astaxanthin were both significantly lower $(P<0.05)$, in both the abdominal segments and pleopods. The rate of degradation of total astaxanthin, and each astaxanthin form, in the abdominal segments and pleopods, were seen to be directly proportional (using linear regression) to the initial content of that form $\left(r^{2}=0.86\right.$, total astaxanthin in abdominal segments; $r^{2}=0.97$, total astaxanthin in pleopods).

A directly proportional relationship between initial $\%$ of esterified astaxanthin and initial total astaxanthin content $\left(r^{2}=0.74\right)$ was seen for the pleopods. A similar but weaker relationship was seen for the abdominal segments $\left(r^{2}=0.43\right)$.

\section{Discussion}

Despite the commercial significance of colour loss of prawns during frozen storage, there is little published information describing astaxanthin content and colour properties of cooked prawns during storage. Bak et al. (1999) investigated astaxanthin content and visual appearance of wild caught and cooked cold-water prawn species (Pandalus borealis) during storage at $-18^{\circ} \mathrm{C}$. The total astaxanthin content was measured in a composite of meat and surrounding shell (head, tail, legs and roe removed), with the initial content being $66 \mathrm{mg} \mathrm{kg}^{-1}$. This was considerably higher than those reported in the abdominal segments for the present work, which may have been due to the higher presence of cartenoid algal species in nutrient-rich cold harvest waters off the coast of Norway for P. borealis compared with tropical pondwaters for black tiger prawns. Despite the higher initial content of astaxanthin in the cold-water prawns, the astaxanthin loss over time was greater in black tiger prawns than observed in $P$. borealis suggesting potential differences in the astaxanthin profile present in each of the prawn species. However, there are other reasons that could explain the different rates of loss including the handling, freezing and storage methods of the prawns. One such difference in the way P. borealis was handled, relative to the prawns in the current study, was that it was frozen with a fresh water glaze, which may have reduced the rate of astaxanthin degradation.
The astaxanthin loss in dried prawn (Penaeus indicus) stored at $4^{\circ} \mathrm{C}$ was $10 \%$ after 4 weeks and reached $45 \%$ after 16 weeks (Niamnuy et al. 2008), which, in terms of \% loss, was higher than that seen in the abdominal segments in the present study, but similar to that in the pleopods. However, the degradation rate in dried prawn stored at $4^{\circ} \mathrm{C}$ (0.60 day $\left.^{-1}, \times 10^{2}\right)$ was similar to the rate in black tiger abdominal segments $\left(0.54\right.$ day $\left.^{-1}, \times 10^{2}\right)$, but lower than the rate in pleopods $\left(1.15\right.$ day $^{-1}$, $\times 10^{2}$ ). Astaxanthin loss in frozen prawns (Bak et al. 1999), dried prawns (Niamnuy et al. 2008) and fish fillets (Gobantes, Choubert \& Gomez 1998), when stored out of the light, is due to oxidation, with storage of frozen $P$. borealis in modified atmosphere packaging (100\% nitrogen gas) significantly reducing astaxanthin degradation (Bak et al. 1999). The in vitro products of astaxanthin auto-oxidation have been shown to be a series of cleavage products with major reaction products including 9-apoastaxanthinone, 11-apoastaxanthinal, 13-apoastaxanthinone and 10-apoastaxanthinal (Etoh, Suhara, Tokuyama, Kato, Nakahigashi, Maejima, Ishikura, Terada \& Maoka 2012).

Astaxanthin content has been described in wild caught black tiger prawns from India (Sachrindra, Bhaskar \& Mahendrakar 2005) and farmed black tiger prawns from Thailand (Menasveta et al. 1993; Okada et al. 1994; Boonyaratpalin et al. 2001), Indonesia (Okada et al. 1994) and Philippines (Okada et al. 1994). Menasveta and colleagues gave no details of how prawns were prepared for analysis but, depending on feeding regime, the content ranged from 11.1 to $44.8 \mathrm{mg} \mathrm{kg}^{-1}$ in shell and from 4.0 to $13.5 \mathrm{mg} \mathrm{kg}^{-1}$, in flesh (Menasveta et al. 1993). In both the shell and flesh the relative percentage of esterified astaxanthin increased with increasing total astaxanthin content, which is similar to what was observed in the present study. Okada and colleagues described the part they extracted as the 'exoskeleton of tails (the edible part)', with astaxanthin content ranging from 2.3 to $33.1 \mathrm{mg} 100 \mathrm{~g}^{-1}$ (Okada et al. 1994). Non-esterified astaxanthin content was the main component (61-65.6\%) in the samples with lower astaxanthin content but was only $30 \%$ in the samples with the highest total astaxanthin content. Okada and colleagues suggested this was indicative of an upper limit of non-esterified astaxanthin accumulation.

Available data for Australian-grown black tiger prawns is from recent work of Tume et al. (2009) 
Table 4 Rate constants $(k$; mean \pm SD, $n=7)$ of degradation of total astaxanthin, and each astaxanthin form, in abdominal segment and pleopod

\begin{tabular}{llll}
\hline $\begin{array}{l}\text { Astaxanthin } \\
\text { form }\end{array}$ & Prawn part & $\begin{array}{l}\boldsymbol{k}\left(\mathbf{d a y}^{-\mathbf{1}},\right. \\
\left.\times 10^{\mathbf{2}}\right)\end{array}$ & $\boldsymbol{r}^{\mathbf{2}}$ \\
\hline Total astaxanthin & Abdominal segment & $0.54 \pm 0.08^{\mathrm{a}}$ & 0.83 \\
Total astaxanthin & Pleopod & $1.15 \pm 0.40^{\mathrm{b}}$ & 0.97 \\
Cis-astaxanthin & Abdominal segment & $0.39 \pm 0.08^{\mathrm{B}}$ & 0.89 \\
Trans-astaxanthin & Abdominal segment & $0.32 \pm 0.06^{\mathrm{B}}$ & 0.79 \\
Mono-esters & Abdominal segment & $0.66 \pm 0.12^{\mathrm{A}}$ & 0.96 \\
Di-esters & Abdominal segment & $0.57 \pm 0.11^{\mathrm{A}}$ & 0.91 \\
Cis-astaxanthin & Pleopod & $0.92 \pm 0.59^{\mathrm{b}}$ & 0.99 \\
Trans-astaxanthin & Pleopod & $0.58 \pm 0.36^{\mathrm{b}}$ & 0.99 \\
Mono-esters & Pleopod & $1.31 \pm 0.63^{\mathrm{a}}$ & 0.98 \\
Di-esters & Pleopod & $1.70 \pm 0.65^{\mathrm{a}}$ & 0.96 \\
\hline
\end{tabular}

Different letters, within each group, indicate statistical significance $(P<0.05)$.

and Wade et al. (2012) who reported the influence of environmental grow-out and background colour on the harvest colour of the prawns. Both studies describe total astaxanthin and astaxanthin profiles in the cephalothorax, abdominal epidermal layer, abdominal exoskeleton and digestive gland, as well the tail (combined abdominal epidermal layer and abdominal exoskeleton). The colour of storage tanks was seen to significantly change the astaxanthin profile in both epidermal layer and exoskeleton of prawns, suggesting a mobility between non-esterified to esterified forms of astaxanthin in a live animal.

It is difficult to directly compare the astaxanthin content between studies due to differences in parts of prawn that were used for extraction, however, the trend identified in the present study, where high initial astaxanthin content showed a predominance of esterified forms, is similar to that reported for other studies on black tiger prawns (Menasveta et al. 1993; Okada et al. 1994). A similar trend was also reported for Litopenaeus vannamei, with feeding regimes that increased total astaxanthin content also increasing the \% of esterified forms in tail muscle samples (Ju et al. 2011). On analysis of the data of Ju et al. (2011), fitted using an exponential function, good correlations were shown between esterified (\%) and total astaxanthin content in both tail muscle $\left(r^{2}=0.73\right)$ and whole abdomen $\left(r^{2}=0.76\right)$.

There was a wide range of astaxanthin content $\left(47-160 \mu \mathrm{g} \mathrm{g}^{-1}\right)$ in initial analysis of pleopods observed in the present study, but there was generally little difference in astaxanthin content after
124-371 days storage. The higher rate of degradation, in more initially concentrated samples, was associated with a higher \% of esterified astaxanthin, which degraded more rapidly than nonesterified astaxanthin. However, the faster degradation of the esterified forms of astaxanthin as compared with the non-esterified forms, observed in every storage trial undertaken, in both pleopods and abdominal segments, was not expected. It had been previously proposed that the non-esterified forms of astaxanthin would be more susceptible to oxidation than esterified astaxanthin forms in dried prawns (Niamnuy et al. 2008; Becerra et al. 2014). However, these studies did not test this hypothesis (only measuring total astaxanthin by visible spectrometry), and no other studies could be found that had examined the oxidation rate of different astaxanthin forms. In the hypodermis, Wade and colleagues have recently shown that esterified astaxanthin is contained in an 'insoluble pellet fraction' but most of the nonesterified astaxanthin is bound to the protein crustacyanin in 'the soluble protein fraction' (Wade et al. 2012). The difference in degradation rates, seen in the present study, may be due to differences in the location and binding of the different astaxanthin forms. The idea of an upper limit of free astaxanthin accumulation during dietary supplementation (Okada et al. 1994), above which astaxanthin is accumulated in esterified form, may suggest that the more recently accumulated esterified astaxanthin would then be lost first during storage.

In the preliminary trial (119 days storage, 2010, see Table 1), abdominal segments of the prawns had higher initial astaxanthin content and greater degradation rates compared to the later trials (sourced 2011 season). It is suggested this may be due to the 2010-harvest prawns being sourced late in the harvest season at which time they would have gained sustained dietary astaxanthin from applied feed and may also have suffered temperature stress. It is recognized that stress induced by any cause (temperature, starvation, predation, disease) results in attempts by the animal to "camouflage' against their background and within a pond environment, and they will typically 'darken' to hide against the pond bottom (Herring 1973; Torrissen, Christiansen, Struksnaes \& Eastermann 1995; Gobantes et al. 1998; Armenta \& GuerreroLegarreta 2009). Feeding regimes, used to boost astaxanthin content in prawns for improved colour and appearance, increase the \% of esterified 
Figure 3 Total astaxanthin content in abdominal segments (a) and pleopods (b) during storage at $-20^{\circ} \mathrm{C}$ for up to 371 days (mean $\pm \mathrm{SD}, n=7$; asterisk indicates value is significantly different $[P<0.05]$ to 0 day).
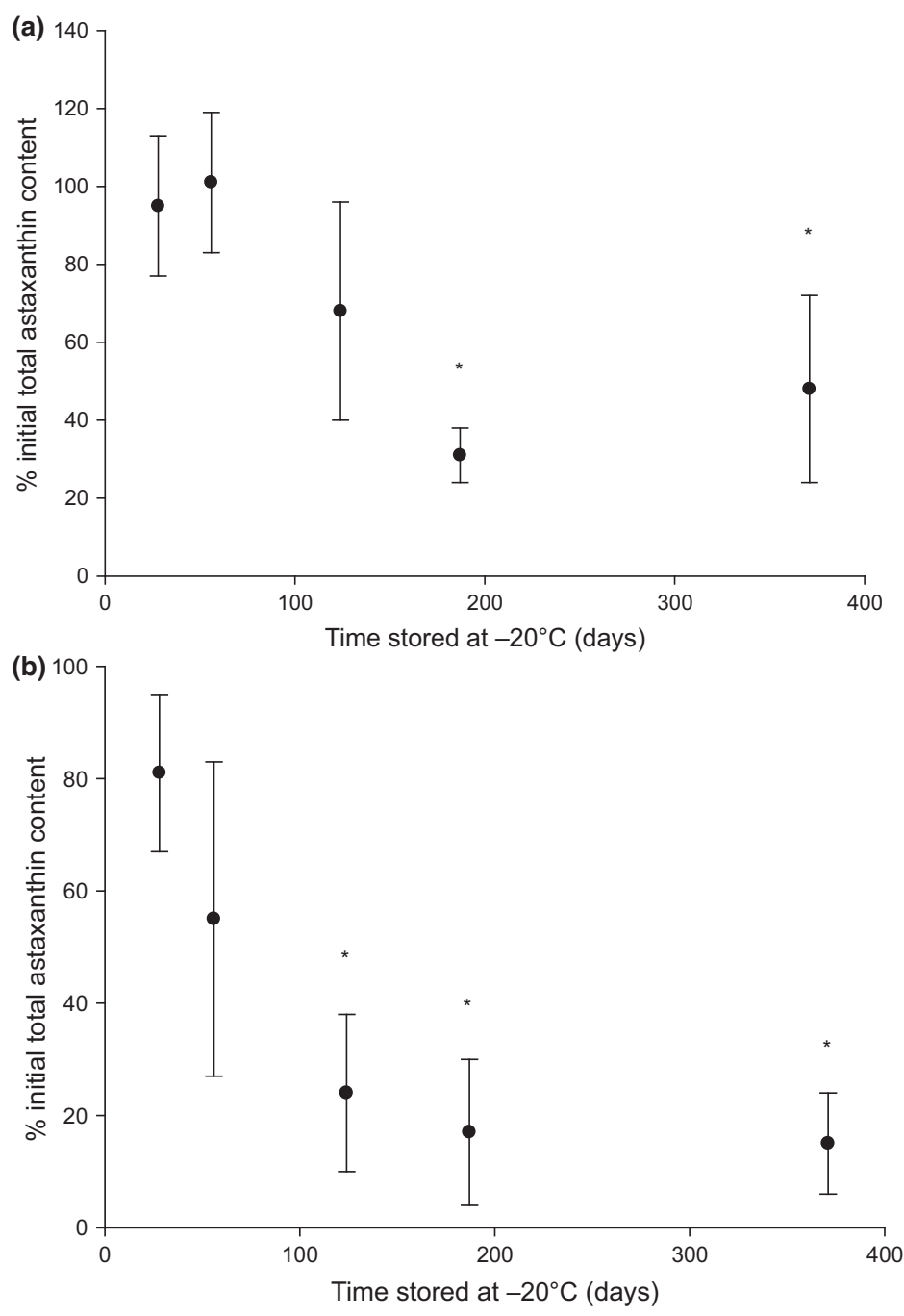

astaxanthin (Menasveta et al. 1993; Okada et al. 1994). As these forms degrade faster than nonesterified forms and higher initial total astaxanthin content was associated with faster degradation of total astaxanthin, when stored at $-20^{\circ} \mathrm{C}$, preservation of the astaxanthin during storage is required to gain the benefit from the feeding regime.

In the 371 days storage trials, the slower decrease of astaxanthin content in abdominal segments relative to the pleopods could be directly related to the relatively lower proportion of esterified forms within the abdominal segments. The rapid loss of astaxanthin in the pleopods compared to abdominal segments was also correlated with greater change in colour parameters. This accords with visual observations that the orange colouration in pleopods of stored tiger prawns fades to yellow hues more rapidly than the body of the prawn. Thus, it is proposed that assessment of pleopod colour is a key monitoring tool for black tiger prawn quality during storage. Future work examining the localization and binding of astaxanthins in the pleopods is also warranted to determine if differences exist between the pleopods and abdominal segments.

Astaxanthin content correlated well with certain colour properties obtained for black tiger prawns, with hue angle and/or a* value appearing to be reasonably good predictors of astaxanthin content in the pleopods during individual storage trials. This is in accord with studies for fresh (Ju et al. 2011) and dried Litopenaeus vannamei (Niamnuy et al. 2008). However, it is noted that the relationship between astaxanthin content and colour appearance of prawns is complex and dependent 

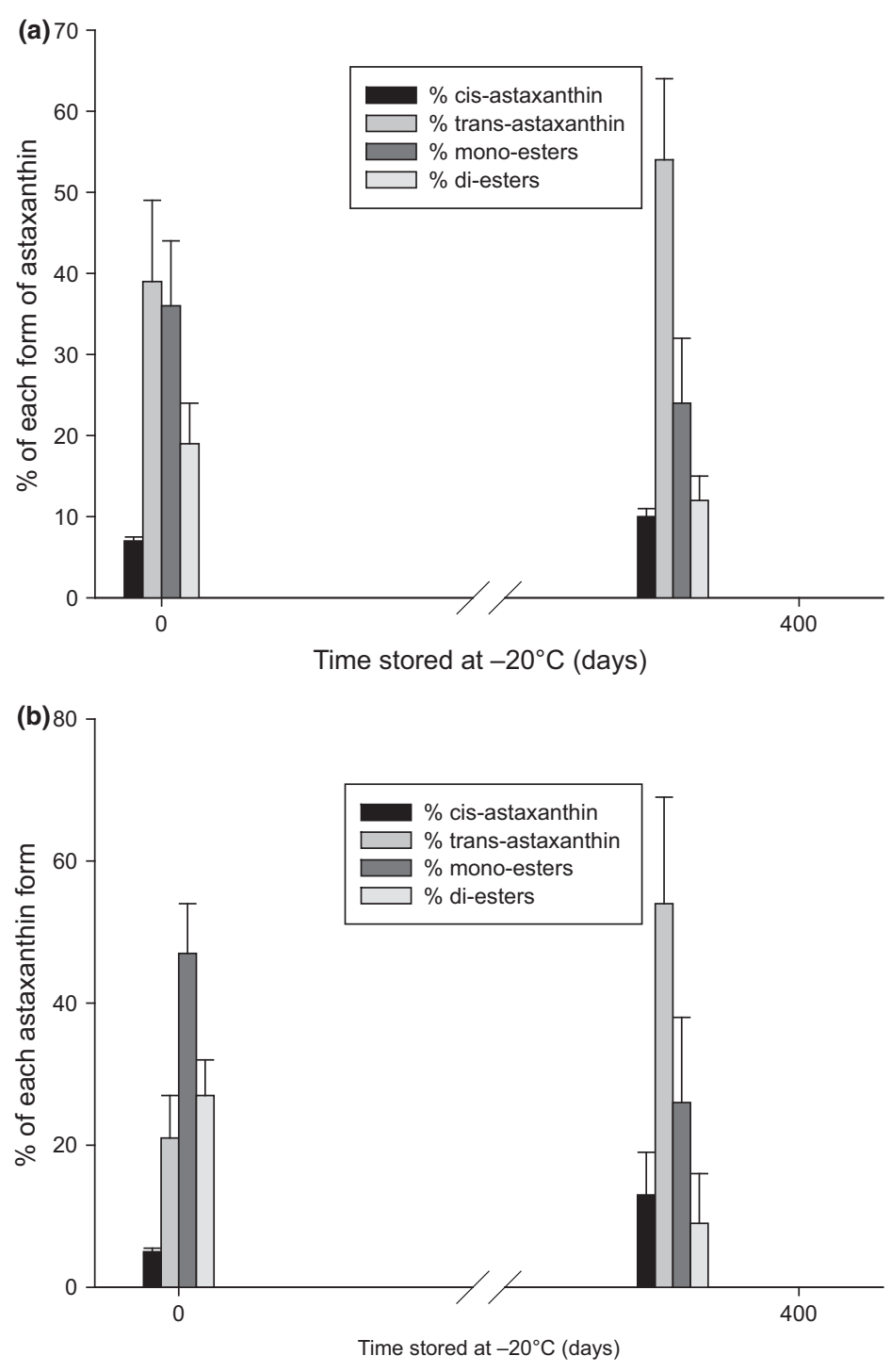

Figure 4 Change in relative proportions of astaxanthin forms in abdominal segments (a) and pleopods (b) during $-20^{\circ} \mathrm{C}$ storage for 371 days (mean $\pm \mathrm{SD}, n=7$ ).

on several factors including mobility between the form and binding of astaxanthin, and a strong direct correlation between astaxanthin content and overall colour, of the hypodermis, does not always occur (Tume et al. 2009; Wade et al. 2012). Black tanked prawns (relative to white tanked prawns) have been shown to have a larger percentage of non-esterified astaxanthin (Tume et al. 2009) and a higher abundance of crustacyanin (Wade et al. 2012), with the crustacyanin-bound, non-esterified astaxanthin in the hypodermis thought to be central to colour changes (Wade et al. 2012). As chromophores are composed of protein bound, non-esterified astaxanthin (Okada et al. 1994), it appears that in P. monodon, astaxanthin is stored as non-esterified astaxanthin to build carotenoproteins, and then excessive astaxanthin amounts are stored as esters (Okada et al. 1994).

This study has determined astaxanthin degradation patterns within both prawn abdominal segments and pleopods, with the latter structures losing astaxanthin and fading in colour more rapidly during frozen storage. As the loss of bright red-orange colour has direct impact on purchase choice at retail level and therefore revenue return for the prawn farming industry, this work has illustrated the importance of using preservation methods for the retention of astaxanthin in black tiger prawns. 


\section{Acknowledgments}

The authors gratefully acknowledge the active support of the Australian Prawn Farmers Association and Aqua Marine Marketing for the open provision of industry information. The generous financial support of the Australian Government through the Australian Seafood Co-operative Research Centre and the Fisheries Research and Development Corporation is fully acknowledged. Warwick Turner's help in setting up the LC-MS system is also acknowledged.

\section{References}

ABARE (2010) Australian Fisheries Statistics, 2009. Australian Bureau of Agricultural and Resource Economics, Australian Goverment, Canberra.

Armenta R.E. \& Guerrero-Legarreta I. (2009) Stability Studies on Astaxanthin Extracted from Fermented Shrimp Byproducts. Journal of Agricultural and Food Chemistry 57, 6095-6100.

Bak L.S., Andersen A.B., Andersen E.M. \& Bertelsen G. (1999) Effect of modified atmosphere packaging on oxidative changes in frozen stored cold water shrimp (Pandalus borealis). Food Chemistry 64, 169-175.

Becerra J.A.H., Flores A.A.O., Valerio-Alfaro G., SotoRodriguez I., Rodriguez-Estrada M.T. \& Garcia H.S. (2014) Cholesterol oxidation and astaxanthin degradation in shrimp during sun drying and storage. Food Chemistry 145, 832-839.

Boonyaratpalin M., Thongrod S., Supamattaya K., Britton G. \& Schlipalius L.E. (2001) Effects of $\beta$-carotene source, Dunaliella salina, and astaxanthin on pigmentation, growth, survival and health of Penaeus monodon. Aquaculture Research 32, 182-190.

Breithaupt D.E. (2004) Identification and quantification of astaxanthin esters in shrimp (Pandalus borealis) and in a microalga (Haematococcus pluvialis) by liquid chromatography-mass spectrometry using negative ion atmospheric pressure chemical ionization. Journal of Agricultural and Food Chemistry 52, 3870-3875.

Chien Y.-H. \& Jeng S.-C. (1992) Pigmentation of kuruma prawn, Penaeus japonicus Bate, by various pigment sources and levels and feeding regimes. Aquaculture 102, 333-346.

Etoh H., Suhara M., Tokuyama S., Kato H., Nakahigashi R., Maejima Y., Ishikura M., Terada Y. \& Maoka T. (2012) Auto-oxidation products of astaxanthin. Journal of Oleo Science 61, 17-21.

Fanning K.J., Martin I., Wong L., Keating V., Pun S. \& O'Hare T. (2010) Screening sweetcorn for enhanced zeaxanthin concentration. Journal of the Science of Food and Agriculture 90, 91-96.

Fujii K., Nakashima H., Hashidzume Y., Uchiyama T., Mishiro K. \& Kadota Y. (2010) Potential use of the astaxanthin-producing microalga, Monoraphidium sp. GK12, as a functional aquafeed for prawns. Journal of Applied Phycology 22, 363-369.

Gobantes I., Choubert G. \& Gomez R. (1998) Quality of Pigmented (Astaxanthin and Canthaxanthin) Rainbow Trout (Oncorhynchus mykiss) Fillets Stored under Vacuum Packaging during Chilled Storage. Journal of Agricultural and Food Chemistry 46, 4358-4362.

Herring P.J. (1973) Depth distribution of the carotenoid pigments and lipids of some oceanic animals. 2. Decapod crustaceans. Journal Marine Biological Association (UK) 53, 539-562.

Ju Z.Y., Deng D.F., Dominy W.G. \& Forster I.P. (2011) Pigmentation of Pacific white shrimp, Litopenaeus vannamei, by dietary astaxanthin extracted from Haematococcus pluvialis. Journal of the World Aquaculture Society 42, 633-644.

Menasveta P., Worawattanamateekul W., Latscha T. \& Clark J.S. (1993) Correction of black tiger prawn (Penaeus monodon fabricius) coloration by astaxanthin. Aquacultural Engineering 12, 203-213.

Niamnuy C., Devahastin S., Soponronnarit S. \& Vijaya Raghavan G.S. (2008) Kinetics of astaxanthin degradation and color changes of dried shrimp during storage. Journal of Food Engineering 87, 591-600.

Okada S., Nureborhan S.A. \& Yamaguchi K. (1994) Carotenoid composition in the exoskeleton of commercial black tiger prawns. Fisheries Science 60, 213-215.

Parisenti J., Beirao L.H., Maraschin M., Mourino J.L., Do Nascimento Vieira F., Bedin L.H. \& Rodrigues E. (2011a) Pigmentation and carotenoid content of shrimp fed with Haematococcus pluvialis and soy lecithin. Aquaculture Nutrition 17, e530-e535.

Parisenti J., Beirão L.H., Tramonte V.L.C.G., Ourique F., da Silveira Brito C.C. \& Moreira C.C. (2011b) Preference ranking of colour in raw and cooked shrimps. International Journal of Food Science \& Technology 46, 2558-2561.

Sachrindra N.M., Bhaskar N. \& Mahendrakar N.S. (2005) Carotenoids in different body components of Indian shrimps. Journal of the Science of Food and Agriculture 85, 167-172.

Torrissen O.J., Christiansen R., Struksnaes G. \& Eastermann R. (1995) Astaxanthin deposition in the flesh of Atlantic salmon, Salmo salar L., in relation to dietary astaxanthin concentration and feeding period. Aquaculture Nutrition 1, 77-84.

Tume R.K., Sikes A.L., Tabrett S. \& Smith D.M. (2009) Effect of background colour on the distribution of astaxanthin in black tiger prawn (Penaeus monodon): effective method for improvement of cooked colour. Aquaculture 296, 129-135.

Wade N.M., Anderson M., Sellars M.J., Tume R.K., Preston N.P. \& Glencross B.D. (2012) Mechanisms of colour adaptation in the prawn Penaeus monodon. The Journal of Experimental Biology 215, 343-350. 